Revista Brasileira de Meteorologia, v.27, n.3, 365 - 375, 2012

\title{
CLASSIFICAÇÃO SINÓTICA DOS CAMPOS DE PRESSÃO ATMOSFÉRICA NA AMÉRICA DO SUL E SUA RELAÇÃO COM AS BAIXAS DO CHACO E DO NOROESTE ARGENTINO
}

\author{
GUSTAVO CARLOS JUAN ESCOBAR ${ }^{1}$ E MARCELO ENRIQUE SELUCHI ${ }^{2}$
}

\author{
${ }^{1}$ Centro de Previsão de Tempo e Estudos Climáticos, Instituto Nacional de Pesquisas Espaciais (CPTEC/ \\ INPE), Cachoeira Paulista, SP, Brasil \\ ${ }^{2}$ Centro de Previsão de Tempo e Estudos Climáticos, Instituto Nacional de Pesquisas Espaciais (CPTEC/INPE), \\ Centro Nacional de Monitoramento e Alertas e Desastres Naturais (CEMADEN), Cachoeira Paulista, SP, Brasil
}

gustavo.escobar@cptec.inpe.br, marcelo.seluchi@cemaden.gov.br

Recebido Maio de 2011 - Aceito Dezembro de 2011

\begin{abstract}
RESUMO
O trabalho apresenta uma classificação dos campos de pressão atmosférica ao nível médio do mar sobre América do Sul, durante as estações de verão e inverno, com o intuito de identificar os principais padrões sinóticos associados à Baixa do Noroeste da Argentina (BNOA) e à Baixa do Chaco $(\mathbf{B C H})$. Para isso, a técnica de Análise de Componentes Principais rotacionadas foi aplicada à serie de reanálises do NCEP para o período 1985-2010. Um segundo objetivo foi determinar as principais frequências de variabilidade relacionadas com a atuação de ambos os sistemas, mediante a utilização do método de Análise Espectral. Os resultados destacam cinco padrões de circulação que representam aproximadamente $80 \%$ dos casos analisados. Durante o verão, a $\mathbf{B C H}$, localizada entre o norte da Argentina, Paraguai, Mato Grosso do Sul e o sul da Bolívia, está associada principalmente com dois padrões sinóticos. A BNOA está associada apenas com um padrão sinótico, similar ao campo médio sazonal. Já no inverno, a BCH não aparece, confirmando seu caráter térmico, gerada principalmente pela liberação de calor latente, produto da convecção observada na área de atuação deste sistema. A BNOA também está associada apenas com um padrão sinótico durante esta estação. Este padrão é similar ao obtido no verão, porém representando menor porcentagem de variância e relacionado principalmente com perturbações sinóticas. A análise de frequência mostra que o modo de circulação associado à $\mathbf{B C H}$ tem um pico de energia em torno dos 17 dias, enquanto que a BNOA apresenta um pico de energia em torno dos 22 dias durante o verão e um relativo aumento da variabilidade sinótica no inverno.
\end{abstract}

Palavras chave: classificação sinótica da pressão atmosférica, BNOA, BCH, componentes principais.

\section{ABSTRACT: SYNOPTIC CLASSIFICATION OF THE ATMOSPHERIC PRESSURE FIELDS OVER SOUTH AMERICA AND ITS RELATION TO THE CHACO AND THE ARGENTINEAN NORTHWEST LOWS}

This work performs a classification of sea level pressure fields for South America during summer and winter, in order to identify the main synoptic patterns associated with the Northwestern Argentinean Low (NAL) and the Chaco Low (CHL). To this purpose, the Principal Component Analysis technique in rotated mode was applied to the NCEP reanalysis for the period from 1985 to 2010. A second objective was to determine the main variability frequencies related to both systems, by employing Spectral Analysis. The results provide five circulation patterns, representative of about $80 \%$ of all cases. During summer, the CHL, located between northern Argentina, Paraguay, Bolivia and southern MS, is mainly associated with two synoptic patterns. The NAL is associated with a single synoptic mode, similar to the seasonal mean field. During winter, the CHL is not recognized, in agreement with its thermal nature related to latent heat released by tropical convection. The NAL is also associated with a unique synoptic mode during this season. This mode is similar to that obtained in the summer and is also related to synoptic scale disturbances but explaining a lesser percentage of variance. The Frequency Analysis show that the circulation mode associated to the CHL has an energy peak at around 17 days, while the NAL has an energy peak around 22 days in summer and a relative increase of synoptic variability in winter.

Keywords: Synoptic classification, NA, CHL, Principal Components 


\section{INTRODUÇÃO}

Durante o verão, uma grande área de baixa pressão se estende, em média, a leste da Cordilheira dos Andes desde a Amazônia até o noroeste da Argentina. Embora os mapas médios elaborados a partir de análises de baixa resolução mostrem um único sistema meridionalmente alongado, existem dois centros independentes e com características diferentes, como pode ser observado na Figura 1, construída a partir das reanálises do modelo Eta/CPTEC (Aravéquia et al., 2008, Herdies et al., 2008) para o período 2000-2004, com resolução de 40km.

A baixa cujo centro se localiza na região do Chaco (entre a Bolívia e o Paraguai) é normalmente conhecida como Baixa do Chaco $(\mathrm{BCH})$. Esta depressão, posicionada em média entorno dos $20^{\circ} \mathrm{S}$ e $60^{\circ} \mathrm{W}$, tem sido pouco estudada na literatura. Recentemente Seluchi e Saulo (2010), através da análise de dois casos particulares, mostraram que a formação da $\mathrm{BCH}$ e da Alta da Bolívia respondem ao aquecimento troposférico provocado principalmente, no caso do verão, pela liberação de calor latente produto da convecção gerada na área de atuação deste sistema. Os fluxos superficiais de calor latente e sensível também possuem um papel importante, já que são os responsáveis diretos do aquecimento das camadas troposféricas mais baixas e, por outro lado, determinam o ciclo diurno da convecção. Durante a primavera, o aquecimento superficial provocado pelo fluxo de calor sensível se torna mais importante, detectando-se também uma influência evidente de perturbações de grande escala (aquecimento por subsidência de grande escala). $\mathrm{A} \mathrm{BCH}$ possui uma estrutura de tipo barotrópica e está imersa numa massa de ar quente, úmida, potencialmente instável e pouco afetada pela atividade transiente (Seluchi et al., 2010). A BCH é um dos sistemas mais característicos da circulação atmosférica em baixos níveis da América do Sul, tendo um papel relevante no funcionamento das "Monções da América do Sul" (Kousky e Kayano,1994, Seluchi e Marengo, 2000). Desta maneira a BCH influencia as condições do tempo no centro-norte da Argentina, sul da Bolívia, no Paraguai, nas Regiões Sul e Sudeste e em parte das Regiões Centro-Oeste e Norte do Brasil.

A Baixa do Noroeste Argentino (BNOA), localizada em torno dos $30^{\circ} \mathrm{S}$ a sotavento da Cordilheira dos Andes, é um sistema relativamente mais conhecido e estudado, que possui uma origem térmica no verão e uma forte influencia orográfica no período de inverno. Por essa razão, às vezes ela também é referenciada como a "Baixa Termo-Orográfica". Os processos de formação e as suas principais características foram abordados principalmente por Lichtenstein (1980), Seluchi et al. (2003) e mais recentemente por Ferreira (2008). A BNOA, devido a sua natureza térmica (vinculada aos intensos fluxos superficiais de calor sensível numa região desértica), tem uma presença quase-permanente no verão, que é só interrompida brevemente após a passagem de sistemas frontais frios. No inverno, a aproximação de cavados de nível superior gera aquecimento através de subsidência orográfica forçada, tornando a BNOA um sistema intermitente. Em ambas as estações, a variabilidade temporal da BNOA está associada a fortes anomalias dinâmicas e termodinâmicas no sudeste da América do Sul, e em particular, na Bacia do Prata (Ferreira, 2008).

Os modos de variabilidade da $\mathrm{BCH}$ e da BNOA são essencialmente desconhecidos. Embora não existam antecedentes específicos na literatura, é muito provável que a variabilidade intrasazonal destes sistemas esteja associada a padrões de circulação atmosférica de maior escala e à atuação simultânea de outros sistemas meteorológicos. A Oscilação Intrasazonal de Madden e Julian (OMJ) (associada com a banda 30-60 dias) e sua relação com a variabilidade da precipitação sobre América do Sul no período de verão têm sido bem estudadas por diversos autores (Kousky e Kayano, 1994, Castro e Cavalcanti,2003). Existem também indícios, como o trabalho de Nogués-Paegle e Mo (1997), que indicam que a BNOA e a $\mathrm{BCH}$ podem estar relacionadas com a atuação da Zona de

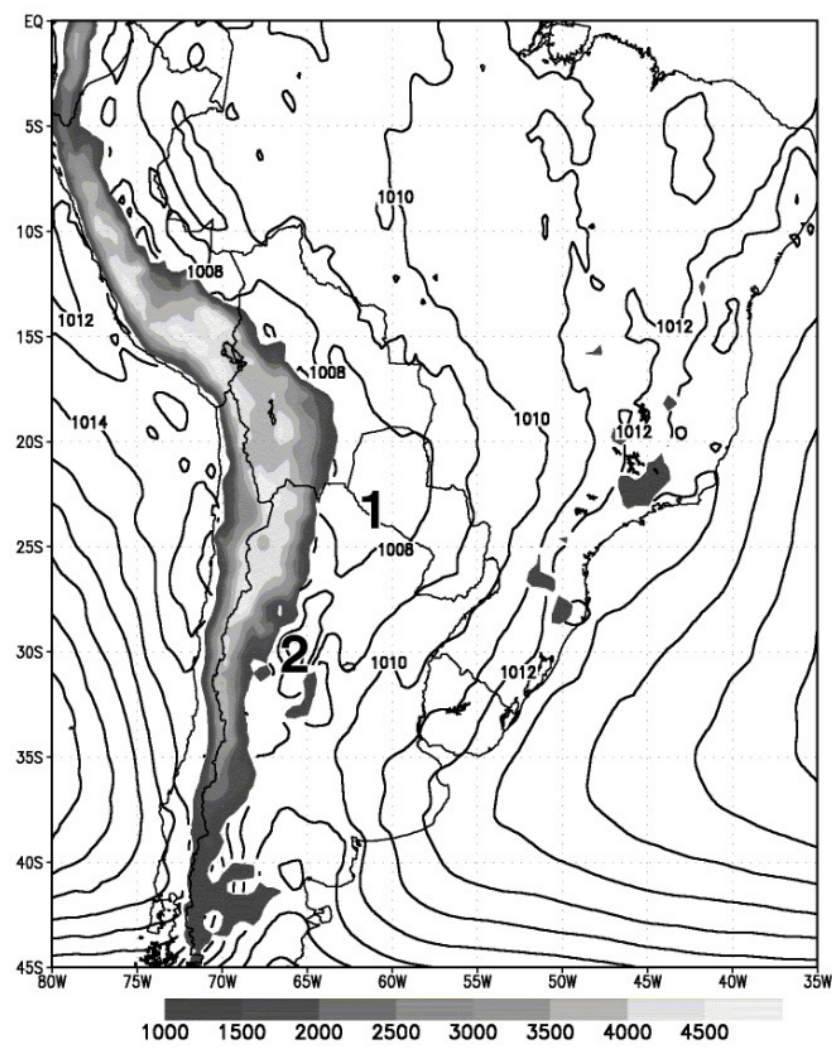

Figura 1 - Campo médio de pressão ao nível médio do mar (hPa) para os meses de verão (dezembro a fevereiro), construído a partir das reanálises do modelo regional Eta/CPTEC para o período 2000-2004. Os tons de cinza indicam elevações acima do nível médio do mar (m). 1: $\mathrm{BCH}, 2$ : BNOA 
Convergência do Atlântico Sul (ZCAS), que ocorre durante a estação chuvosa e cuja variabilidade é modulada pela OMJ. A $\mathrm{BCH}$, devido a sua relação com as monções da América do Sul, é também potencialmente à influência das oscilações intrasazonais. Por outro lado, é sabido que a BNOA é fortemente influenciada pelas perturbações de escala sinótica, especialmente no inverno, enquanto que atuação da $\mathrm{BCH}$ nessa estação do ano não está documentada. $\mathrm{O}$ entendimento dos modos de variabilidade e da sua frequência é de grande importância prática, sendo que, como foi enfatizado anteriormente, a atuação da $\mathrm{BNOA}$ e da $\mathrm{BCH}$ gera fortes impactos no tempo e no clima da região economicamente mais ativa da América do Sul.

Este trabalho tem como objetivo principal identificar os principais padrões sinóticos associados à $\mathrm{BNOA}$ e à $\mathrm{BCH}$, e determinar as principais frequências de variabilidade relacionadas com ambos os sistemas durante o verão e o inverno.

Na seção 2 são apresentados os dados e a metodologia utilizada, a seção 3 mostra os resultados da classificação sinótica e a análise da variabilidade intrasazonal relacionada com ambos os sistemas de baixa pressão. A seção 4 apresenta uma síntese dos principais resultados obtidos neste trabalho.

\section{MATERIAL E MÉTODOS}

\subsection{Dados}

Para a análise dos campos de circulação em superfície foram usados os dados de pressão ao nível médio do mar para os meses de verão e inverno, provenientes da reanálise do NCEP/NCAR (National Center for Environmental Prediction/ National Center for Atmospheric Research) para o período 1986 a 2010 (Kalnay et al., 1996). O verão e o inverno foram definidos a partir dos trimestres de dezembro a fevereiro e de junho a agosto, respectivamente.

\subsection{Análise de Componentes Principais (ACP)}

Para a determinação dos principais modelos ou padrões de circulação associados à presença da $\mathrm{BNOA}$ ou da $\mathrm{BCH}$ foi realizada uma classificação sinótica dos campos de pressão ao nível do mar. A metodologia utilizada foi a Análise de Componentes Principais (ACP) com uma matriz de correlação em Modo - T (Green, 1978; Richman, 1986).

Existem dois tipos de ACP utilizados para analisar a variável observada, tanto no espaço quanto no tempo. O primeiro chamado Modo-S está relacionado com a análise de séries temporais correspondentes a pontos no espaço. Isto significa que a variável estatística analisada corresponde a séries temporais de uma variável meteorológica, que pode ser considerada contínua no espaço e no tempo. Por outro lado, o
Modo-T pode ser aplicado para classificar campos atmosféricos espaciais em diferentes tempos (Compagnucci e Salles, 1997; Escobar e Bischoff, 1999; Escobar et. al., 2004; Escobar, 2007). Neste caso, a variável estatística analisada corresponde ao campo espacial da variável meteorológica em questão. Desta maneira, identificam-se subgrupos ou tipos de campos com a mesma estrutura espacial. Os dados são dispostos em formato de matriz onde as colunas são determinadas pelos campos espaciais correspondentes a tempos consecutivos. As filas desta matriz de entrada são determinadas pelos pontos de grade. Isto significa que cada coluna representa um campo de pressão atmosférica ao nível do mar. Segundo Richman (1983), o Modo-T é uma ferramenta muito útil para sintetizar e reproduzir padrões de circulações, quantificando sua frequência e mostrando os períodos de tempo neles dominantes.

Após a aplicação da ACP foi feita a rotação Varimax, retendo dez componentes principais (CPs) para ambas as estações do ano. Para a determinação do número de componentes rotacionadas utilizou-se a regra do autovalor 1.0 (Richman et. al, 1992).

Após a obtenção das CPs, foram selecionadas situações meteorológicas altamente correlacionadas com cada uma das CPs. Para a determinação destes casos foram utilizadas as series temporais dos fatores de peso (também conhecidos pelo seu nome em inglês: «factor loadings»), que representam as correlações entre cada variável (situação meteorológica real) e cada componente principal (Richman, 1986). A análise da série de «factor loadings» permite determinar a representatividade das componentes principais como situações sinóticas reais, onde valores próximos a 1 representam situações meteorológicas similares às das componentes principais obtidas (Harman, 1976; Cattel, 1978).

\subsection{Análise de variabilidade}

Para estudar a variabilidade da $\mathrm{BNOA}$ e da $\mathrm{BCH}$ foi feita uma análise de frequência das principais oscilações atmosféricas, associadas aos padrões sinóticos relacionados à ambas as baixas. Para isto, foi aplicado o método de Análise Espectral (Jenkins e Watts, 1969) às series dos "factors loadings" de cada uma das três primeiras componentes principais, obtidas durante o verão e o inverno.

A utilização do cálculo do espectro de potência permite analisar o comportamento de uma série temporal em função das periodicidades ou quase-periodicidades contidas nela.

Neste estudo calculou-se a série média dos factors loadings construída a partir dos 25 anos de informação utilizada. Para tal fim, aplicou-se o método de Jones (1964) à série de dados de cada ano, e à série assim obtida foi aplicada a Análise Espectral. O espectro de potência obtido desta maneira permite 
identificar os períodos ou quase-períodos contidos na série, com especial ênfase naquelas frequências que se encontram em fase na maioria dos anos analisados. Desta forma, pode-se pensar em processos nos quais a atmosfera, em distintos anos, produz uma modulação nestas frequências em datas parecidas.

\section{RESULTADOS}

\subsection{Classificação sinótica do verão e do inverno}

A aplicação da Análise de Componentes Principais identificou cinco padrões em superfície que explicaram 79,7\% e $82,98 \%$ da variância total no verão e inverno, respectivamente.

A Tabela 1 mostra as porcentagens da variância explicada e porcentagens da variância acumulada por cada componente principal. A partir destes resultados pode-se observar que durante o verão existe uma maior variabilidade em relação ao inverno, já que as cinco CPs do verão representam menor porcentagem de variância acumulada $(79,7 \%)$ do que as cinco do inverno $(82,9 \%)$.

A análise das componentes de peso (ou "factor loadings") permite avaliar a representatividade dos padrões como situações sinóticas reais. Valores próximos a 1 representam situações meteorológicas similares aos padrões obtidos (Harman, 1976; Cattel, 1978). As primeiras cinco componentes de peso mostraram valores maiores que 0,7 . Isto significa que os padrões teóricos (CPs) e as situações sinóticas reais têm configurações similares. As CPs restantes não foram consideradas neste trabalho já que explicaram menos de $4 \%$ da variância total. Esses padrões também representam situações meteorológicas reais, porém menos frequentes.

$\mathrm{Na}$ Figura 2 observam-se as cinco CPs (painéis superiores) e casos observados (painéis inferiores) altamente correlacionados com elas durante o verão.

O primeiro Padrão ( $\mathrm{CP} 1)$ e o caso real altamente correlacionado (Figuras 2a e 2d, respectivamente) mostram a presença de um centro de baixa pressão, aproximadamente entre o extremo norte da Argentina (Província de Formosa), Paraguai,

Tabela 1 - Porcentagens da variância explicada e da variância acumulada pelas cinco primeiras CPs durante o verão (coluna do meio) e inverno (coluna da direita).

\begin{tabular}{|c|c|c|}
\hline CPs & VAR. (\%) / CUM. VAR (\%) & VAR. (\%) / CUM. VAR (\%) \\
\hline 1 & 26,2 / 26,2 & 28,4 / 28,4 \\
\hline 2 & $25,4 / 51,6$ & $26,5 / 54,9$ \\
\hline 3 & $13,3 / 64,9$ & $12,1 / 67,0$ \\
\hline 4 & $10,7 / 75,6$ & $10,8 / 77,8$ \\
\hline 5 & $4,1 / 79,7$ & $5,1 / 82,9$ \\
\hline
\end{tabular}

sul da Bolívia e o centro-sul de Mato Grosso do Sul (MS). Nota-se que ao sul deste sistema de baixa pressão, há um centro anticiclônico pós-frontal associado com uma frente fria oceânica, aproximadamente na altura do litoral do Estado do Paraná. É possível inferir que a porção sul da baixa pressão possui um vínculo direto com a frente, porém sua posição mais ao norte permite associá-la com a $\mathrm{BCH}$. Os anticiclones subtropicais do Pacífico Sul (ASPS) e do Atlântico Sul (ASAS) aparecem localizados aproximadamente em $40^{\circ} \mathrm{S}$ e $23^{\circ} \mathrm{S}$, respectivamente. Devido à situação sinótica predominante, a ASPS encontra-se localizada ao sul da sua posição climatológica.

O segundo Padrão (CP2) e o caso real associado (Figuras $2 \mathrm{~b}$ e 2e) mostram uma configuração muito semelhante ao campo médio de verão (vide Figuras 1a e 3c de Satyamurty et al., 1998) . Eles permitem identificar uma área de baixa pressão alongada, se estendendo a leste dos Andes desde latitudes altas até o sul da Bolívia. Nota-se que o caso real mostra uma baixa fechada de $1004 \mathrm{hPa}$ localizada a leste da Cordilheira dos Andes, na posição típica da BNOA. Mais ao sul pode-se identificar um cavado oceânico a leste da Patagônia, possivelmente associado com uma frente fria. Neste padrão sinótico, a frente fria sobre o continente estaria localizada imediatamente ao sul da BNOA, de acordo com a configuração climatológica discutida em Ferreira (2008). O ASAS mostra seu núcleo principal mais ao sul, aproximadamente em $40^{\circ} \mathrm{S}$, devido em parte à presença de uma área de baixa pressão relativa localizada próxima a costa de Santa Catarina. Esta depressão relativa, que pode se configurar na forma de cavado ou de baixa é frequentemente observada durante o verão, em concordância com os resultados de Sinclair (1995), Hoskins e Hodges (2005), Reboita et al. (2010) e Guia (2010). Segundo a classificação de ciclones de Hart (2003), baseada na estrutura dinâmica e termodinâmica do sistema, a maioria destes ciclones são extratropicais fracos, porém em certas ocasiões eles podem adquirir características subtropicais ou raramente tropicais. Estes ciclones, formados durante, o verão estão mais associados a mecanismos termodinâmicos do que dinâmicos (Iwabe e Da Rocha, 2009, Reboita, 2008). Este modelo padrão, em função do posicionamento, orientação e extensão do cavado, também pode estar associado a eventos de ZCAS (Escobar e Da Costa, 2005), que ocorrem levemente mais ao sul da sua posição normal (Sanches, 2002).

A terceira Componente Principal (CP3) e o respectivo caso altamente correlacionado (Figuras $2 \mathrm{c}$ e $2 \mathrm{f}$ ) permitem identificar duas áreas de baixa pressão, uma mais intensa e bem definida localizada entre o norte da Argentina, Paraguai, oeste de MS e sul da Bolívia, e outra mais fraca no oceano Attântico a leste da Região Sudeste. A baixa continental está claramente associada à $\mathrm{BCH}$, devido a seu posicionamento e à ausência de forçantes sinóticas, enquanto que a área ciclônica oceânica corresponde a uma região ciclogenética preferencial na América 

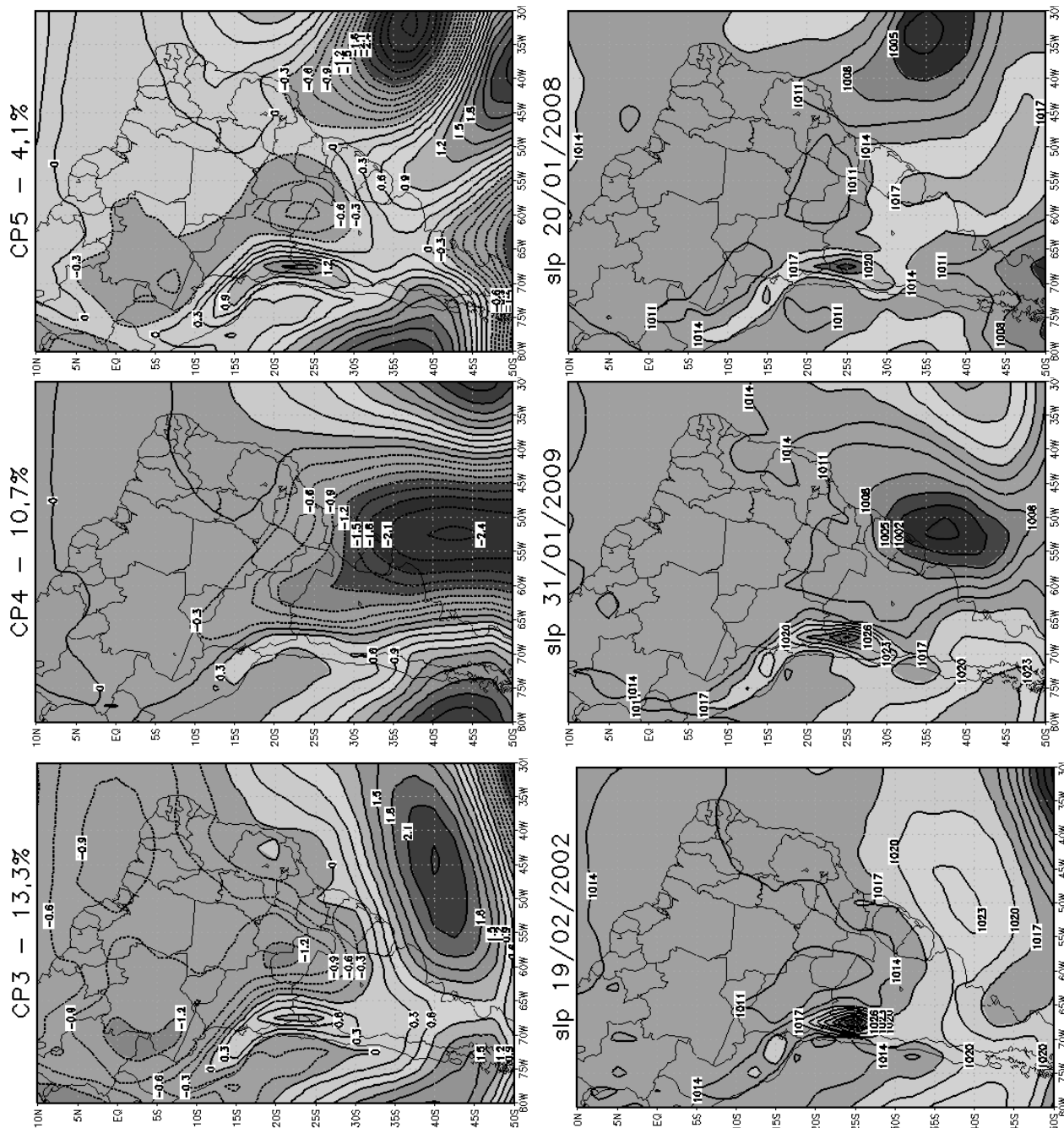

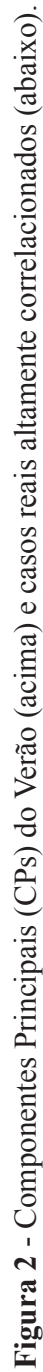
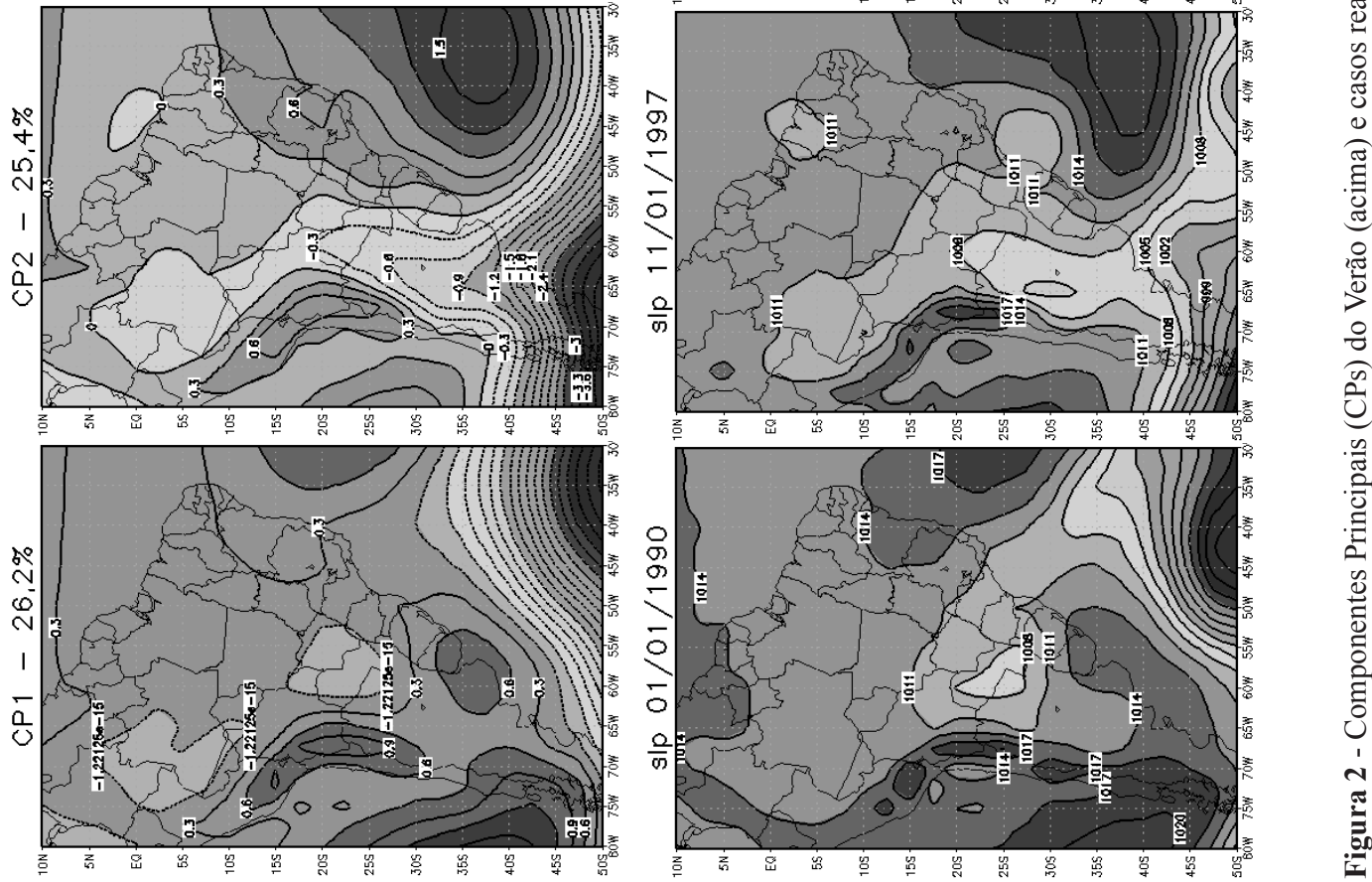
do Sul durante o verão (Sinclair, 1995; Hoskins e Hodges, 2005; Reboita et al., 2010; Guia, 2010), muitas vezes favorecida pela liberação de calor latente resultante da ocorrência de chuva (Guia, 2010) e fluxo de calor latente do oceano para a atmosfera (Reboita, 2008). Em certas ocasiões, este sistema de baixa pressão pode-se apresentar apenas como um cavado ou como uma frente estacionária em frontólise, e em ambos os casos pode estar associados a episódios de ZCAS (Carvalho et al., 2004; Escobar e Da Costa, 2005). Esta depressão pode ficar deslocada mais para leste ou nordeste, porém sempre embebida dentro da ASAS que geralmente encontra-se deslocada mais ao sul da a sua posição climatológica, devido às características de tipo bloqueio. O quarto Padrão (CP4) e o caso real correspondente (Figuras $2 \mathrm{~g}$ e $2 \mathrm{i}$ ) mostram uma área de baixa pressão fechada e meridionalmente alongada, se estendendo desde o oceano Atlântico até o sul da Região Sul. Essa situação sinótica pode estar associada com uma onda frontal, cujo ramo frio se estende até o Estado do Rio Grande do Sul (RS) e nordeste da Argentina. Neste caso, tanto a BNOA quanto a $\mathrm{BCH}$ ficam "mascaradas" pela maior profundidade da baixa principal.

A quinta Componente Principal (CP5) e seu caso altamente correlacionado (Figuras $2 \mathrm{~h}$ e $2 \mathrm{j}$ ) mostram uma área de baixa pressão fechada localizada aproximadamente entre o nordeste da Argentina, Paraguai, MS e sul da Bolívia, na região que climatologicamente ocupa a $\mathrm{BCH}$. No oceano Atlântico pode-se observar outra área ciclônica mais intensa, associada à presença de uma onda frontal localizada aproximadamente em $35^{\circ} \mathrm{S}, 32^{\circ} \mathrm{W}$. O ramo frio deste ciclone extratropical se estende em direção à costa da Região Sudeste do Brasil e o anticiclone pós-frontal aparece de forma alongada, se estendendo desde o oceano até o sul da Região Sul do Brasil. Este padrão sinótico é pouco frequente no verão uma vez que representa $4 \%$ da variância total dos dados.

Na Figura 3 observam-se as cinco CPs (painéis acima) e casos observados altamente correlacionados com elas (painéis abaixo) durante o inverno.

O primeiro Padrão ( $\mathrm{CP} 1)$ e o caso real altamente correlacionado (Figuras 3a e 3c) são semelhantes ao campo médio de pressão para o período de inverno (vide Figura 1c e 3c de Satyamurty et al., 1998). Eles mostram uma área de baixa pressão alongada se estendendo desde a Patagônia até o Paraguai, que se localiza imediatamente ao norte de um cavado menos pronunciado e imerso num intenso fluxo de oeste, característico desta época do ano. Tal cavado representa a posição média das frentes frias mais frequentes no inverno. É possível identificar os anticiclones subtropicais do Pacífico (ASPS) e do Atlântico (ASAS) Sul, aproximadamente na posição climatológica para esta época do ano (vide Fig 3C, 1c, Satyamurty et al., 1998).
No inverno, a BNOA se forma devido à interação da atividade baroclínica com a Cordilheira dos Andes. O balanço negativo de radiação inibe a formação deste sistema e sua presença está relacionada com a passagem de perturbações sinóticas (Ferreira, 2008), que são mais frequentes nesta estação do ano. Desta maneira, a BNOA passa a ter um caráter intermitente.

A segunda Componente Principal (CP2) e o caso associado (Figuras3b e 3d) correspondem à passagem de um sistema frontal frio, aproximadamente na altura da Região Sudeste do Brasil, com um anticiclone pós-frontal se estendendo desde o sul do continente sul-americano até o interior das Regiões Sudeste e Centro-Oeste. Este padrão também foi identificado durante o verão através da CP1 (vide Figura 2), porém apresentando a frente com características mais oceânicas e o anticiclone pós-frontal mais enfraquecido. Pode-se observar que durante o verão, este padrão sinótico está relacionado com a presença da $\mathrm{BCH}$. No entanto, durante o inverno este sistema não aparece, muito provavelmente em decorrência da sua característica térmica.

O terceiro Padrão (CP3) e o caso real altamente correlacionado (Figura 3c e 3f) mostram uma forte incursão de ar frio sobre o continente sul-americano, similar ao encontrado por Escobar (2007), que fez uma classificação sinótica das incursões de ar frio sobre a cidade de São Paulo. Pode-se observar um intenso anticiclone pós-frontal centrado aproximadamente em $39^{\circ} \mathrm{S}$ e $54^{\circ} \mathrm{W}$, abrangendo a maior parte da América do Sul, que normalmente está associado a eventos de queda significativa de temperatura no sul da região amazônica, conhecidos como "friagens" (Marengo et al., 1997).

A quarta Componente Principal (CP4) e o respectivo caso altamente correlacionado (Figuras $3 \mathrm{~g}$ e 3i) mostram uma área de baixa pressão alongada diagonalmente, se estendendo desde o oceano Atlântico Sul em direção ao sul da Bolívia. Este sistema pode estar associado a eventos ciclogenéticos sobre o nordeste da Argentina, Paraguai, Uruguai e o sul da Região Sul do Brasil.

O quinto Padrão (CP5) e o caso real correspondente (Figuras $3 \mathrm{~h}$ e 3j) mostram uma ampla área de baixa pressão se estendendo desde o oceano Pacífico próximo da costa do Chile até o sul da Bolívia. Esta área apresenta dois centros, um sobre o Oceano Pacífico e outro no centro da Argentina, sendo um resultado típico do avanço de perturbações ciclônicas através da Cordilheira dos Andes (Seluchi e Lichtenstein 1992, Gan e Rao 1994), assim como de processos frontogenéticos no interior do continente. Devido à posição das áreas de baixa pressão e às características de tipo transiente surgidas das CP4 e CP5 não é possível identificar à BNOA. No caso da CP5, esse padrão poderia corresponder a um processo ciclogenêtico (similar ao descrito por Seluchi e Saulo 1998), cujo ciclo de vida inclui, pelo menos 24 horas antes, o desenvolvimento da BNOA. 

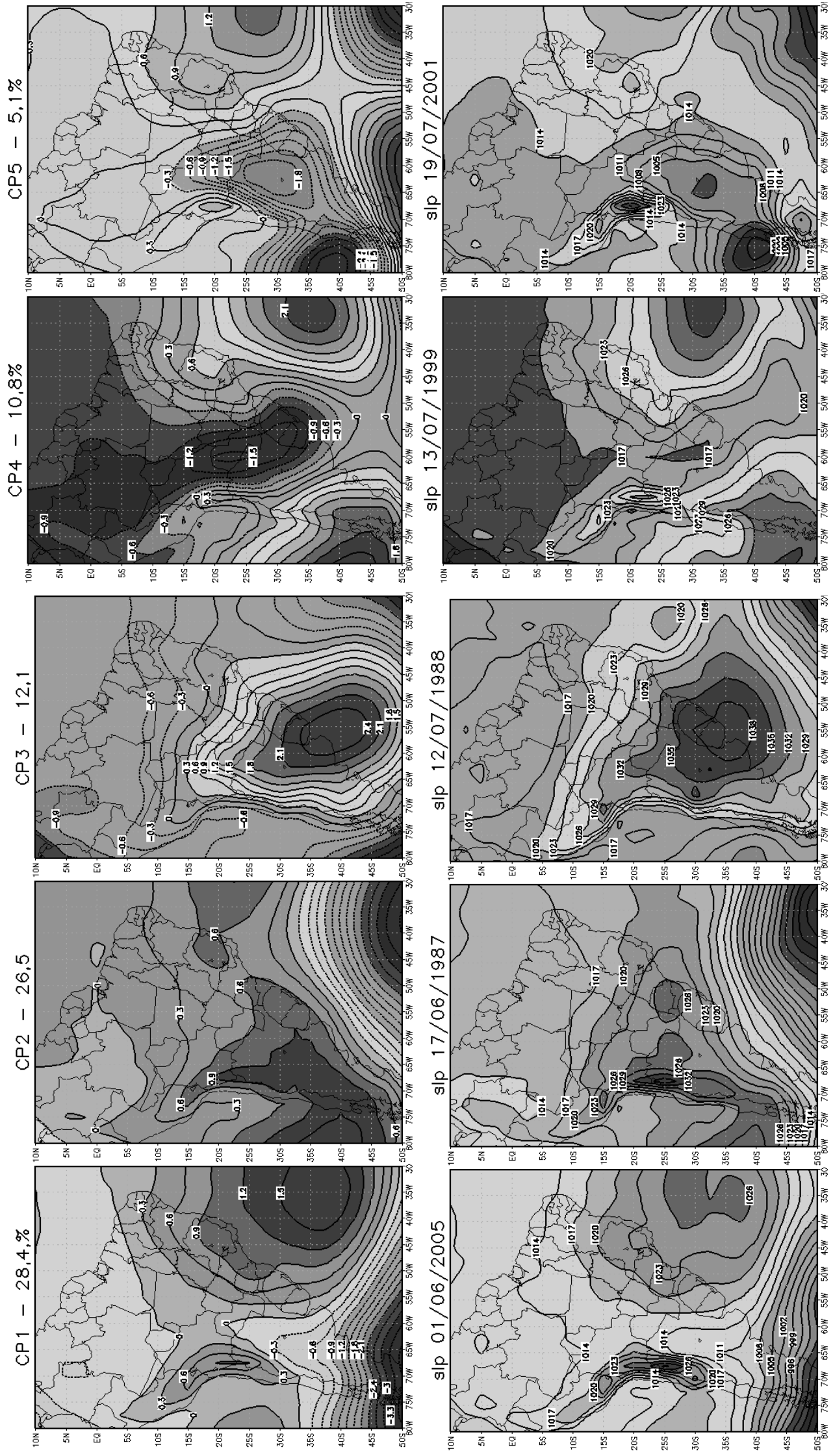

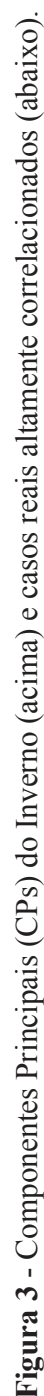




\subsection{Variabilidade intrasazonal}

As Figuras 4 e 5 mostram a análise espectral para as três principais CPs relacionadas ao verão (Figura 2) e inverno (Figura 3), respectivamente.

Durante o verão (Figura 4) o maior pico de energia é representado pela CP1 (Figura 2a), com períodos que oscilam em torno dos 15 dias, representando oscilações atmosféricas de escala maior que a sinótica. Como foi descrito na seção 3.1, este padrão de circulação em superfície está associado com a presença da $\mathrm{BCH}$, de uma frente fria oceânica aproximadamente na altura de Santa Catarina e atuação da ZCAS. O pico de energia em torno dos 17 dias pode estar sendo influenciado pelos episódios de ZCAS, que geralmente persistem por mais de 4 dias com um período de recorrência maior que o correspondente à escala sinótica.

Durante o inverno, a CP2 (Figura 3b), mostra um padrão de circulação parecido à $\mathrm{CP} 1$ de verão (vide Figura 2a), porém sem a presença da $\mathrm{BCH}$ e com a atuação do anticiclone pós-frontal mais intenso. Durante esta época do ano, como é de esperar, este modelo de circulação não está associado com episódios de ZCAS. O espectro de potência apresenta um máximo principal em torno dos 13 dias e dois picos secundários em torno dos 9 e 6 dias, respectivamente (Figura 5). O máximo de potência secundário correspondente aos 6 dias representa oscilações atmosféricas de escala sinótica. Durante o verão

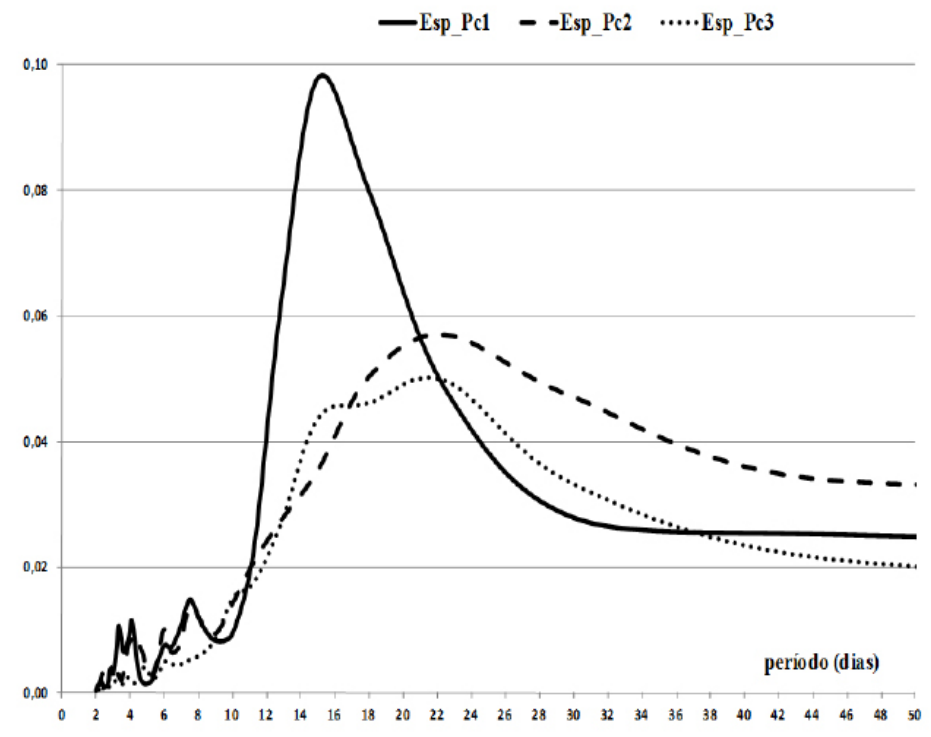

Figura 4 - Espectro de Potência dos 3 primeiros CPs para o verão.

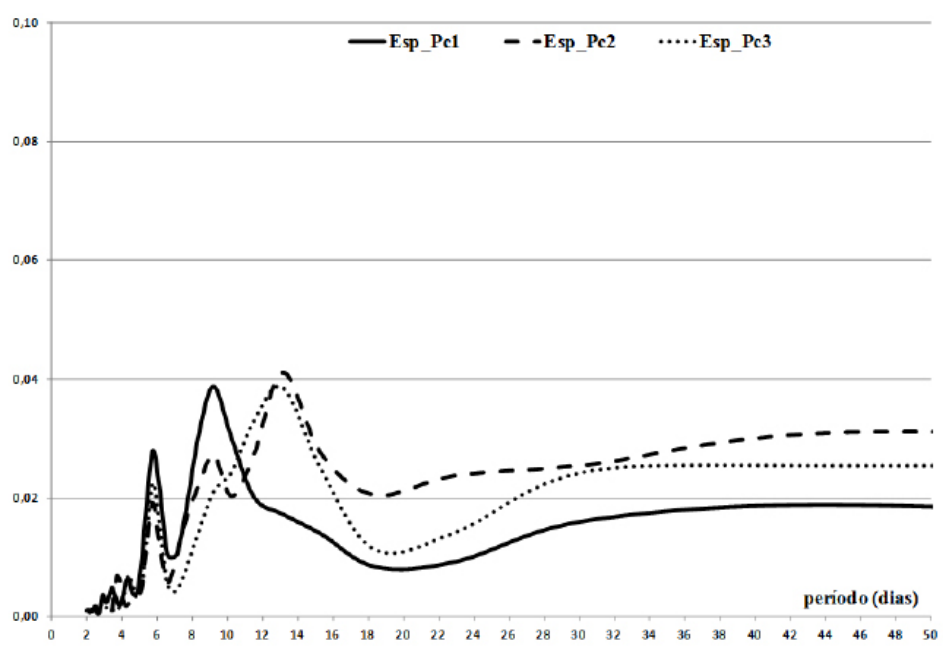

Figura 5 - Espectro de Potência dos 3 primeiros CPs para o inverno. 
(CP1 (Figura 2a) este pico em torno de 6 dias é praticamente desprezível (Figura 4), indicando, como é de esperar, uma menor frequência de sistemas frontais.

$\mathrm{O}$ padrão sinótico, associado à presença da BNOA identificado tanto no verão (CP2, Figura $2 b$ ) quanto no inverno (CP1, Figura 3a), também apresenta espectros de energia diferentes entre ambas as estações. Durante o verão (Figura 4), este modelo de circulação apresenta um pico de energia significativo em torno dos 22 dias, porém com um espectro de potência bastante suavizado se estendendo para períodos maiores. Nota-se também que, para períodos inferiores aos 12 dias, o espectro de potência se reduz drasticamente. Pode-se observar um pico secundário em torno dos 8 dias, porém muito fraco. Durante o inverno (Figura 5) este modelo de circulação apresenta dois máximos significativos de energia, porém bem menos intensos do que durante o verão. Nota-se um máximo principal de espectro de potência em torno dos 9 dias e um máximo secundário em torno dos 5 dias. Este modelo de circulação em superfície representa o campo bárico mais frequente ao longo do ano, sendo que durante o inverno, devido à maior baroclinia, este padrão se torna mais recorrente.

A CP3 obtida durante o verão também está associada à presença da $\mathrm{BCH}$ (Figura 2c) e mostra um espectro de potência similar àquele obtido para a CP2 de inverno (Figura 5), isto significa um máximo principal em torno dos 22 dias.

No inverno, a CP3 está relacionada com episódios de fortes incursões de ar frio (Figura 3c) e seu espectro de potência apresenta dois máximos em torno de 6 e 13 dias.

Em resumo, pode-se dizer que durante o verão, devido à menor baroclinia, as ondas atmosféricas respondem a uma escala de frequências mais baixas em relação ao inverno. Nesta última época do ano, as ondas atmosféricas principais estão relacionadas a escalas de tempo que oscilam entre os 6 e 13 dias. Entretanto, no verão as flutuações atmosféricas principais estão em torno dos 16 e 22 dias, aproximadamente.

\section{CONCLUSÕES}

A análise da variabilidade dos campos de circulação em superfície, correspondente às estações de verão e inverno, para o continente sul-americano, mostra que existem diferentes padrões sinóticos associados à presença da $\mathrm{BNOA}$ e da $\mathrm{BCH}$.

Para ambas as estações do ano foram encontradas cinco padrões principais que representam aproximadamente $80 \%$ dos casos (Tabela 1). Durante o verão, aproximadamente $70 \%$ desses casos estão associados à presença da $\mathrm{BNOA}$ e/ou da $\mathrm{BCH}$. Esse resultado indica que ambos os sistemas dominam a circulação nos baixos níveis nesta época do ano. Já durante o inverno, apenas aproximadamente $40 \%$ dos casos estão relacionados com a $\mathrm{BNOA}$, sendo que $10 \%$ destes casos mostram à $\mathrm{BNOA}$ com uma forte influência dinâmica, associada à passagem de perturbações transientes. A $\mathrm{BCH}$ não pode ser identificada durante o inverno, o que está de acordo com os resultados da literatura, que a citam como um sistema de características térmicas, formado pelos fluxos superficiais de calor latente e sensível.

Os padrões de circulação em superfície mais típicos de verão (CP1 e CP2) explicam mais do $50 \%$ da variância total (Tabela 1), e representam os padrões sinóticos mais frequentes relacionados com a $\mathrm{BCH}$ e a $\mathrm{BNOA}$, respectivamente. A CP2 corresponde a um padrão sinótico bastante similar ao campo médio do verão, onde se podem identificar claramente os anticiclones subtropicais na sua posição climatológica e um cavado localizado no interior da Argentina. Este cavado representa com maior probabilidade a posição média da BNOA, como foi descrito na seção 3.1.

A CP1 e CP3 representam situações meteorológicas associadas com a $\mathrm{BCH}$, localizada aproximadamente entre o norte da Argentina, Paraguai, Mato Grosso do Sul e o sul da Bolívia.

A CP5 também descreve um padrão sinótico associado com a $\mathrm{BCH}$, porém com uma variância explicada inferior ao $5 \%$, indicando que este modelo de circulação é pouco frequente.

Tanto a $\mathrm{CP} 1$ quanto a $\mathrm{CP} 5$ mostram a $\mathrm{BCH}$ junto à presença de um sistema frontal no oceano na altura do Sul e do Sudeste, respectivamente. Mesmo assim, a influência dinâmica sobre a $\mathrm{BCH}$, produzida por esses transientes, aparenta ser bem fraca.

A CP1 e CP3 de verão representam os modelos mais frequentes relacionados com eventos de ZCAS, ambos associados com a $\mathrm{BCH}$ que contribui para direcionar o fluxo quente e úmido (Jato de Baixos Níveis -JBN) da região amazônica para parte das Regiões Sul e Sudeste do Brasil. A CP1 descreve um padrão sinótico relacionado com uma frente fria na altura de São Paulo, entretanto a CP3 mostra um padrão sinótico com um anticiclone localizado em $40^{\circ} \mathrm{S}, 45^{\circ} \mathrm{W}$ e um cavado no oceano na altura de São Paulo e do Rio de Janeiro. Este sistema de baixa pressão relativo favorece a convergência de massa e de umidade desde o interior do continente até o Atlântico. Em situações de ZCAS o padrão sinótico relacionado com a CP3 persiste por vários dias configurando-se uma situação de tipo bloqueio. Desta maneira, o anticiclone localizado no oceano adquire maior intensidade e aparece posicionado ao sul da sua posição climatológica. Padrões sinóticos similares à $\mathrm{CP} 1$ e à CP3 foram encontrados por Escobar e Da Costa (2005), que fez uma classificação sinótica de episódios de ZCAS.

Durante o inverno pode-se observar apenas um padrão sinótico, representado pela $\mathrm{CP} 1$, associado com a BNOA, que nesta estação do ano está mais relacionada com a passagem de perturbações transientes. Os padrões sinóticos, representados 
pelas $\mathrm{CP} 4$ e CP5, também estão associados a um centro de baixa pressão, porém com características exclusivamente dinâmicas.

Durante o verão, o modelo de circulação associado exclusivamente à $\mathrm{BCH}(\mathrm{CP} 1)$, mostra um pico de energia em torno dos 15 dias, representando oscilações atmosféricas de escala maior do que a sinótica. A CP3, também associada à presença da $\mathrm{BCH}$ durante o verão, mostra um pico de energia em torno dos 22 dias, porém de menor intensidade em relação à $\mathrm{CP} 1$, descrita anteriormente.

Durante o inverno, este mesmo padrão sinótico (CP2) (sem a presença da $\mathrm{BCH}$ ), mostra em ordem crescente três picos de energia correspondente aos períodos de 6,9 e 13 dias, respectivamente. A presença de oscilações atmosféricas de alta frequência durante o inverno está relacionada diretamente com o aumento da baroclinicidade, que se traduz em um aumento na frequência de sistemas frontais. Durante o verão nota-se uma diminuição na frequência de sistemas frontais e, em consequência, um aumento nas oscilações atmosféricas de baixa frequência.

Os resultados apresentados neste trabalho mostram, pela primeira vez na literatura, que a $\mathrm{BCH}$ é um sistema exclusivo da estação quente, e que está associada a oscilações de escala maior que a sinótica. Esse fato resulta coerente com a sua atuação no âmbito das monções da América do Sul, que são normalmente afetados pela variabilidade intra-sazonal (Vera et al., 2006, Ferreira e Gan, 2011). Da mesma forma, a BNOA possui uma variabilidade semelhante à da $\mathrm{BCH}$ no verão, provavelmente vinculada às oscilações do jato subtropical que, por sua vez, respondem parcialmente às variações de intensidade e posição da Alta da Bolívia. Durante o inverno, a BNOAé mais intermitente e ligada às perturbações transientes que interagem com a Cordilheira dos Andes. Por essa razão, os picos de energia são menores que no verão e mostram o aumento relativo no espectro correspondente à escala sinótica.

Finalmente, este trabalho conclui através da técnica de $\mathrm{ACP}$, que a $\mathrm{BCH}$ e a $\mathrm{BNOA}$ são sistemas diferentes que não atuam nas mesmas épocas do ano.

\section{REFERÊNCIAS BIBLIOGRÁFICAS}

ARAVEQUIA, J. A. ; HERDIES, D. L. ; SAPUCCI, L. F. ; ANDREOLI, R. V. ; FERREIRA S.H.S. ; GONÇALVES, L. G.G. . Reanálise Regional 2000-2004 sobre a América do Sul com o Modelo RPSAS/ETA: Descrição do Experimento e dos Produtos Derivados. Boletim da Sociedade Brasileira de Meteorologia, v. 32, p. 71-77, 2008.

CARVAlHO V. ; JONES, C. ; LIEBMANN, B. The South Atlantic Convergence Zone: Persistence, Form, Extreme Precipitation and Relationships with Intraseasonal Activity. Journal of Climate, v. 17, n. 1, p. 88-108, 2004.
CASTRO, C.C.; CAVALCANTI, I. F.A. 2003. Intraseasonal modes of variability affecting the SACZ. VII international Conference on Southern Hemisphere Meteorology and Oceanography, Anais..., Wellington, New Zealand, 2003, p. 24-28.

CATTELL, R. The scientific use of factor analysis: in Behavioral and Life Sciences. Plenum Press. New York amd London, 1978.

COMPAGNUCCI, R.; SALLES, M.A. Surface Pressure Patterns during the year over Southern South America. International Journal of Climatology, v. 17, n. 6, p. 635-653, 1997

ESCOBAR, G. C. J. Padrões sinóticos associados a ondas de frio na cidade de São Paulo. Revista Brasileira de Meteorologia, v. 22, p. 241-254, 2007.

ESCOBAR, G. C. J.; BISCHOFF, S. Criterio de detección de irrupciones de aire frío en la region central de Argentina a partir de descensos interdiurnos de temperatura. Meteorologica, v. 26, n. 1 y 2, p. 57-68, 2001.

ESCOBAR, G.; BISCHOFF, S. Meteorological situations associated with significant temperature falls in Buenos Aires: an application to the daily consumption of residential natural gas. Meteorlogical Applications, v. 6, n. 3, p. 253-260, 1999.

ESCOBAR, G. C. J.; COMPAGNUCCI, R. H.; BISCHOFF, S. A. Sequence Patterns of $1000 \mathrm{hPa}$ and $500 \mathrm{hPa}$ geopotential height fields associated with cold surges in Buenos Aires. Atmosfera, v 12, n. 2, p. 69-89, 2004.

ESCOBAR, G. C. J.; DA COSTA, I C. Situações meteorológicas associadas a episódios da Zona de Convergência do Atlântico Sul (ZCAS). IX Congresso Argentino de Meteorología, Anais..., 2005.

FERREIRA, L.: Causas y variabilidad de la Depresión del Noroeste Argentino e Impactos sobre los Patrones Regionales de Circulación. Tese de Doutorado. Universidade de Buenos Aires. 177 pp. 2008.

FERREIRA, S.; GAN, M. Intraseasonal variability in the South American monsoon system. Atmospheric Science Letters, v.12, n. 3, p. 253-260, 2011.

GAN MA, RAO VB: The influence of the Andes Cordillera on transient disturbances. Monthly Weather Review, v. 122, p. 1141-1157, 1994.

GUIA, C. V. F. Análises das características sinóticas das trajetórias dos ciclones extratropicais que atuam na América do Sul e Vizinhanças. 2010. 105 p. Dissertação (Mestrado em Meteorologia) - Instituto Nacional de Pesquisas Espaciais, São José dos Campos. (2010) Disponível em: $<$ http://urlib.net/8JMKD3MGP7W/38LS7UE $>$.

GREEN, P. Analysing Multivariate Data. The Dryden Press. Illinois, U.S.A, 519, 1978.

HARMAN, H. Modern Factor Analysis. The University of Chicago Press Chicago, IL, 1976. 
HART, R. E. A cyclone phase space derived from thermal wind and thermal asymmetry. Monthly Weather Review, v. 131, p. 585-616, 2003.

HERDIES, D. L. ; ARAVEQUIOA, J. A.; FERREIRA S.H.S.; ADREOLIi, R. V. ; SAPUCCI L. F. ; MATTOS, J. G. Z.. A Assimilação de Dados no CPTEC/INPE. Boletim da Sociedade Brasileira de Meteorologia, v. 32, p. 57-64, 2008.

HOSKINS, B. J; HODGES, K. I. A new perspective on the southern hemisphere storm tracks. Journal of Climate, v. 18, p. 4108-4129, 2005.

IWABE, C.; DA ROCHA, R. An event of stratospheric air intrusion and its associated secondary surface cyclogenesis over the South Atlantic Ocean. Journal of Geophysical Research, 114, D09101, 15 pp., 2009.

JENKINS, J. M; WATTS, D. G. Spectral Analysis and its applications. Hoden-day series in time series analysis, 2 edicion, San Francisco, Cambridge, London, Amsterdam, 1969.

JONES, R.H. Spectral analysis and linear prediction of meteorological time series. Journal of Applied Meteorology, v. 3, p. 45-52, 1964.

KALNAY, E. et al. The NCEP/NCAR 40-year reanalysis Project. Bulletin of the American Meteorological Society, v. 77, n. 3, p. 437-471, 1996.

KOUSKY, V. E.; KAYANO, M. T. Principal modes of outgoing longwave radiation and $250 \mathrm{hPa}$ circulation for the South American setor. Journal of Climate, v. 7, p. 1131-1142, 1994.

LICHTENSTEIN, E. R. La Depresión del Noroeste Argentino. Tese de Doutorado em Meteorologia, Universidade de Buenos Aires, Buenos Aires. 1980.

MARENGO, J.A.; NOBRE, C.A.; Culf, A.D. Climatic Impacts of "Friagens" in forested and deforested areas of the Amazon Basin. Journal of Applied Meteorology, 36: 1553-1566, 1997.

NOGUÉS-PAEGLE, J.; MO, K. C. Alternating wet and dryconditions over South America during summer. Monthly Weather Review, v.125, p. 279-291, 1997.

REBOITA, M. S. Ciclones extratropicais sobre o Atlântico Sul: Simulação climática e experimentos de sensibilidade. 2008. 359 pág. Tese de Doutorado em Meteorologia Instituto de Astronomia, Geofísica e Ciências Atmosféricas IAGUSP, Universidade de São Paulo, São Paulo, 2008.

REBOITA, M. S.; NIETO, R.; GIMENO, L.; DA ROCHA, R. P.; AMBRIZZI, T.; GARREAUD, R.; KRUGEL, L. F. Climatological features of cutoff systems in the Southern Hemisphere. Journal of Geophysical Research, 115, p. D17104, 2010.
RICHMAN, M. Specification of complex modes of circulation with T-mode factor analysis. In: II INTERNATIONAL CONFERENCE ON STATISTICS AND CLIMATE, 1983, Lisbon. Anais..., National Institute of Meteorology and Geophysics, p. 511-518, 1983.

RICHMAN, M. Rotation of Principal Components. Journal of Climate, v. 6, n. 3, p. 293-335, 1986.

RICHMAN, M.; ANGEL, J.; GONG, X. Determination of Dimensionality in Eingenanalysis. In: V INTERNATIONAL MEETING ON STATSTICAL CLIMATOLOGY, 6, Canada. Anais...:. p. 229-235, 1992.

SANCHES, M.B. Análise sinótica da Zona de Convergência do Atlântico Sul (ZCAS) utilizando-se a técnica de composição. 2002. Dissertação de Mestrado no Instituto Nacional de Pesquisas Espaciais, São José dos Campos, 2002.

SATYAMURTY, P.;NOBRE, C., SILVA DIAS, P.L., Meteorology of The Southern Hemisphere. Chapter 3C, v. 27, n. 49, p. 119-139, American Meteorological Society, 1998.

SELUCHI, M.E.; SAULO, C, Mecanismos físicos associados à formação da Baixa do Chaco, XVI Congresso Brasileiro Meteorologia, Belém, PA, Anais..., 2010.

SELUCHI, M.E.; SAULO, C , GARREAUD, R.D., Baixa termo-orográfica e Baixa do Chaco: dois sistemas diferentes?, XVI Congresso Brasileiro Meteorologia, Belém, PA, Anais..., 2010.

SELUCHI, M.E.; MARENGO, J.A. Tropical-extratropical Exchange of air masses during Summer and Winter in South America:Climatic Aspects and extreme events. International Journal of Climatology, v. 20, p. 11671190, 2000.

SELUCHI, M.E.; SAULO, C. A.; NICOLINI, M.; SATYAMURTY, P. The Northwestern Argentinean Low: a study of two typical events. Monthly Weather Review, $\mathbf{v}$, v. 131, p. 2361-2378, 2003.

SELUCHI, M. E. ; SAULO, A C. Possible mechanisms yielding and explosive coastal cyclogenesis over South America: experiments using a Limited Area Model. Australian Meteorological Magazine, v. 47, p. 309-320, 1998.

SELUCHI, M. E. ; LICHTENSTEIN, E. R. Campos medios asociados a sistemas ciclónicos y anticiclónicos sobre la Argentina. Geoacta, Argentina, v. 19, n. 2, p. 125-136, 1992.

SINCLAIR, M. A. Climatology of cyclogenesis for the southern hemisphere. Monthly Weather Review, v. 123, p. 16011619, 1995.

VERA, C., W. et al. Toward a Unified View of the American Monsoon Systems, Journal of Climate -Special Section, 19, 4977-5000, 2006. 